\title{
Day of surgery cancellation rates in urology: Identification of modifiable factors
}

\author{
Robert J. Leslie, MD; $;^{*}$ Darren Beiko, MD, FRCSC; ${ }^{*}$ Janet van Vlymen, MD; ${ }^{\dagger}$ D. Robert Siemens, MD, FRCSC ${ }^{* \$ t}$
}

`Departments of Urology, Queen's University, Kingston, ON; †Anesthesiology and Perioperative Medicine, Queen's University, Kingston, ON; §Oncology, Queen's University, Kingston, ON; 汭iomedical and Molecular Sciences, Queen's University, Kingston, ON

Cite as: Can Urol Assoc J 2013;7(5-6):167-73. hitrp://dx.doi.org/10.5489/cuaj.12020. Epub 2012 Sept 10.

\section{Abstract}

Objective: Day-of-surgery cancellations have a negative effect on operating room $(\mathrm{OR})$ resources, as well as on patient satisfaction and perception of quality of care. Given increasing wait times in a universal healthcare system and the nature of urological surgery in our aging population, it should be a priority to identify modifiable risks of OR cancellations to assure timely and efficient delivery of care. We explore the rate and reasons for elective surgery cancellations in a Canadian urological practice.

Methods: We evaluated the rate and reason of urological surgery cancellation at a single academic institution, prospectively collected in our centre's Operating Room Scheduling Office System (ORSOS) database. Documented reasons for cancellations were divided into 3 components: (1) structural factors (e.g., no hospital bed); (2) patient factors (e.g., patient unwell); and (3) process factors (e.g., scheduling error). Rates and reasons for cancellations were compared to those of General Surgery and Gynecology. The documented reasons for cancellation in the ORSOS database were confirmed or extended by chart review and interviews with a subset of cancelled patients.

Results: Between 2005 and 2009, 1544 out of 19141 (8.07\%) elective surgical cases were cancelled within the three surgical specialties (general surgery, gynecology and urology); urology had the highest average rate of $9.53 \%$. Non-oncological cases represented a higher percentage of cancelled cases $(15 \%, p<0.001)$ and overall rates varied significantly over time in urology compared to the other surgical specialties. Potentially modifiable, processrelated causes were by far the most common reason for cancellation $(58.5 \%)$ and "standby" cases were a common cause of overall cancellation rates. Patient interviews confirmed the emotional and financial impact of cancellation; there was no overwhelming concern that clinical outcomes were negatively affected.

Conclusions: This contemporary exploration of cancelled urological cases is consistent with previous reports, although variable over time and dependent on definitions used. Potentially modifiable, process-related factors appear to be most frequently associated with cancellation, although more thorough and detailed documentation is required to further mitigate inefficient OR use. We suggest that all OR cancellations should be considered to be adverse incidents to be monitored by institutions in a systematic fashion.

\section{Introduction}

Surgical wait time in Canada is the most visible and yet contentious quality of care indicator in our universal healthcare system and is inter-related with all three components of Donabedian's framework: structure, process and patient outcome. ${ }^{1-3}$ Despite much attention paid to pre-operative surgical preparation, day of surgery cancellations remain a major cause of inefficient use of operating room (OR) time and a drain on finite healthcare resources. ${ }^{4-6}$ Cancellations also create a financial burden for patients in addition to potential emotional stress and a negative impact on perception of quality of care..$^{7-10}$ Monitoring cancellation rates and indentifying modifiable causes should be a priority for all stakeholders to assure the timely and efficient delivery of surgical care.

International studies have documented day-of-surgery cancellation rates as high as $13 \%$ for elective surgery and many jurisdictions have instituted limited interventions to decrease these rates, ${ }^{11,12}$ including the introduction of monitoring software ${ }^{13}$ and charging patients directly for missed appointments. ${ }^{14}$ Targeting interventions in focused areas, such as manipulation of case sequence or initiating preoperative evaluations closer to the OR date, ${ }^{15,16}$ appear to be ineffective given the wide-ranging reasons for cancellations. Although it is reported that most cancellations may be avoidable, ${ }^{4,6,17-19}$ a consistent and standardized method of documenting contributing factors is lacking. Although reasons for cancellation are generally patient- or hospitalinitiated, ${ }^{4,17}$ we have categorized these at our institution as structure-related (e.g., no hospital bed), patient-related (e.g., patient unwell) or process-related (e.g., scheduling error) based on Donabedian's quality of care framework. ${ }^{3}$

Cancellation rates vary not only between different hospital types and sizes, ${ }^{1}$ but also depends significantly on surgical 
Leslie et al.

specialty and individual surgeons. ${ }^{4,5,19,20}$ Comparatively high cancellation rates have been reported in specific services, such as urology and otolaryngology, ${ }^{19-21}$ perhaps due to a bias of those with complex and variable case durations. ${ }^{20}$ Given the documented increase in wait times in urology, ${ }^{9}$ further investigation in a contemporary urological setting appears justified given the nature and scope of our specialty in an aging population. The objective of this study was to identify the rate and reasons for elective surgery cancellations in a Canadian urological practice and to explore the ability of available administrative data to categorize potentially modifiable factors.

\section{Methods}

To more fully understand our findings in the context of other hospitals in our region, we first compared our hospital's reported cancellation rates to others contained in an administrative dataset available through Ontario's Operating Room Benchmark Collaborative (ORBC) over the 12-month period of April 1, 2009 to March 31, 2010. The criteria used to compare our centre to peer hospitals included: acute teaching hospital (any number of beds and ORs), academic centre, trauma centre (all levels), oncology program and open heart program. The ORBC data demonstrated only a slightly higher cancellation rate for our institution $(6.6 \%)$ compared to that of other profile-matched Ontario hospitals (5.5\%). Unfortunately, variations in data collection and definitions of reasons for cancellations, as well as a lack of case detail with such administrative data, make it difficult to compare reasons for cancellations between institutions.

Following ethics approval from the Queen's University institutional review board, our centre's Operating Room Scheduling Office System (ORSOS) database was used to examine cancellation rates of all electively booked urological surgery over a 5-year period between January 1, 2005 and December 31, 2009. Surgery cancellation was defined as any operation on the OR list, printed the day before surgery, which then did not proceed. Emergency cases were excluded, but cases listed as "standby patient" were taken into account. "Standby" patients were listed by the surgical service if estimated time of booked cases was greater than time available predicted by the ORSOS system or at the discretion of the surgeon. Rates and reasons for day-of-surgery cancellations were also compared to those in gynecology and general surgery.

Explanations for cancellations are identified and coded by the daily operational triage nurse in the OR and documented in the ORSOS system. With the exception of holidays and minimal vacation and sick-days, a single charge nurse was responsible for codifying all surgery cancellations. There are 37 separate choices for coding a case cancellation (Appendix 1). This method of documentation was developed and revised in collaboration by nurses, surgeons and OR administration staff and has been used since 2000. In an attempt to confirm and further detail the cause of a particular cancellation, we collected data from the OR nurse triage notes and we performed a chart review to identify further information from the anesthesiologist or surgeon's notes. The chart review was only feasible for the last 2 years of the study, between January 1, 2008 and December 31, 2009, when electronic scanning of documentation was introduced.

To better appreciate potential modifiable risk factors and how they change over time and between specialties, we categorized the 37 different coded reasons for surgery cancellation into three groups: (1) patient-related; (2) processrelated; and (3) structure-related. Examples of patient-related cancellations were "patient refused/cancelled procedure" and "patient too ill for surgery." Every attempt was made to ensure these factors were not secondary to incomplete preoperative preparation, which then would have been coded differently. Structure-related cancellations included those related to hospital constraints or uncontrollable factors, such as weather and included "crisis cancellation," "emergency case inserted" and "no bed/step-down bed available." We considered process-related cancellations as those which were due to the organization and facilitation of perioperative care and scheduling, including "anesthesiologist late," "incorrectly booked," "patient not NPO," "room running late" and "surgeon running late."

Finally, standardized phone interviews were conducted with cancelled urological patients booked for surgery between January 2009 and January 2010. Interviews were performed at least 5 months after cancellations to evaluate each patient's experience and satisfaction with the process using open-ended questions and a quantitative survey. These closed-ended questions, each with a 5-point Likert scale, were developed after a literature search and consultation with stakeholders on the healthcare team.

\section{Calculations and statistics}

The surgical cancellation rate was calculated as follows: [number of cases cancelled] / [number of cases cancelled + number of elective cases completed]. Data were presented as rates per calendar year. Descriptive statistics for the closed-ended questions in the patient interview were presented as mean \pm standard deviation $(\mathrm{SD})$. For ease of reporting, agreement scores for the closed-ended questions included the first and second score (strongly agree, agree) in the 5-point Likert scale. Inferential statistics used to compare cancellation rates included Student's two-sample t-test ${ }^{22}$ for continuous data or Fisher's exact test and $\chi 2$ test for dichotomous data. One-way ANOVA was used to compare rates of cancellations over time. GraphPad Prism was used for statistical analysis. 


\section{Results}

The ORSOS database for the three surgical services (gynecology, general surgery, urology) identified 19141 booked elective cases, of which 1544 (8.0\%) were cancelled over the 5-year period of time. Of the three specialties examined, urology had the highest 5-year average cancellation rate at $9.5 \%$ (430/4512). Gynecology had the lowest at $6.8 \%$ and general surgery at $8.2 \%$ (Fig. 1a). Rates appeared to vary with time, especially for the urology service with a peak of $13.4 \%$ and its nadir at $7.3 \%$ in the most recent year; these fluctuations, however, were not statistically significant $(p=0.162$, one-way ANOVA).

Cancellation rates were dependent on the inclusion or exclusion of "standby" cases listed on the elective booking list, particularly for the urology service (Fig. 1b, $p<0.001$ ). Rate of cancellation differed significantly based on surgical indication (Table 1). Non-oncological cases were preferentially cancelled (15\%) compared to genitourinary cancer surgery $(5 \%)$.

Causes of surgical cancelations divided into structure-, process- and patient-related factors are shown for each specialty over the 5 -year period (Table 2). Over 5 years for all three specialties, structure-related causes (Fig. 2) accounted for $22.8 \%$ of OR cancellations, compared to only $19.6 \%$ of patient-related cases (Fig. 3). Structure-related cases of cancellations were most common in general surgery, most often due to a higher number of urgent cases displacing elective case load during the OR day. Although structureand patient-related factors associated with OR cancellations may have been identified preoperatively or modified with a significant increase in resources, process-related causes are likely more easily targeted to affect change. We found that these process-related cases were by far the most common among the three specialties at $57.6 \%$ (Fig. 4), which is similar to other reports. ${ }^{20}$ Interestingly, the reduction in cancellation rates in the urology service was associated mostly with improvements in the process-related factors.

Of the 50 cases cancelled in the 2009 calendar year, 29 patients were successfully contacted and interviewed (58\% response rate). However, $26 \%$ (13/50) of all cases had no updated contact information and only $16 \%$ with valid information were not interviewed ( 3 did not want to participate and 5 were unreachable by phone). The interview results showed that about half of the patients contacted were notified of the need for cancellation less than 60 minutes prior to, or after, their scheduled OR time. Most interview respondents said they were made aware of the reason for the cancellation, although $4(14 \%)$ reported no information was given and in another $4(14 \%)$ cases, the reasons given were not corroborated by the administrative data. Out of the 29 patients interviewed, 20 (69\%) were notified in person,

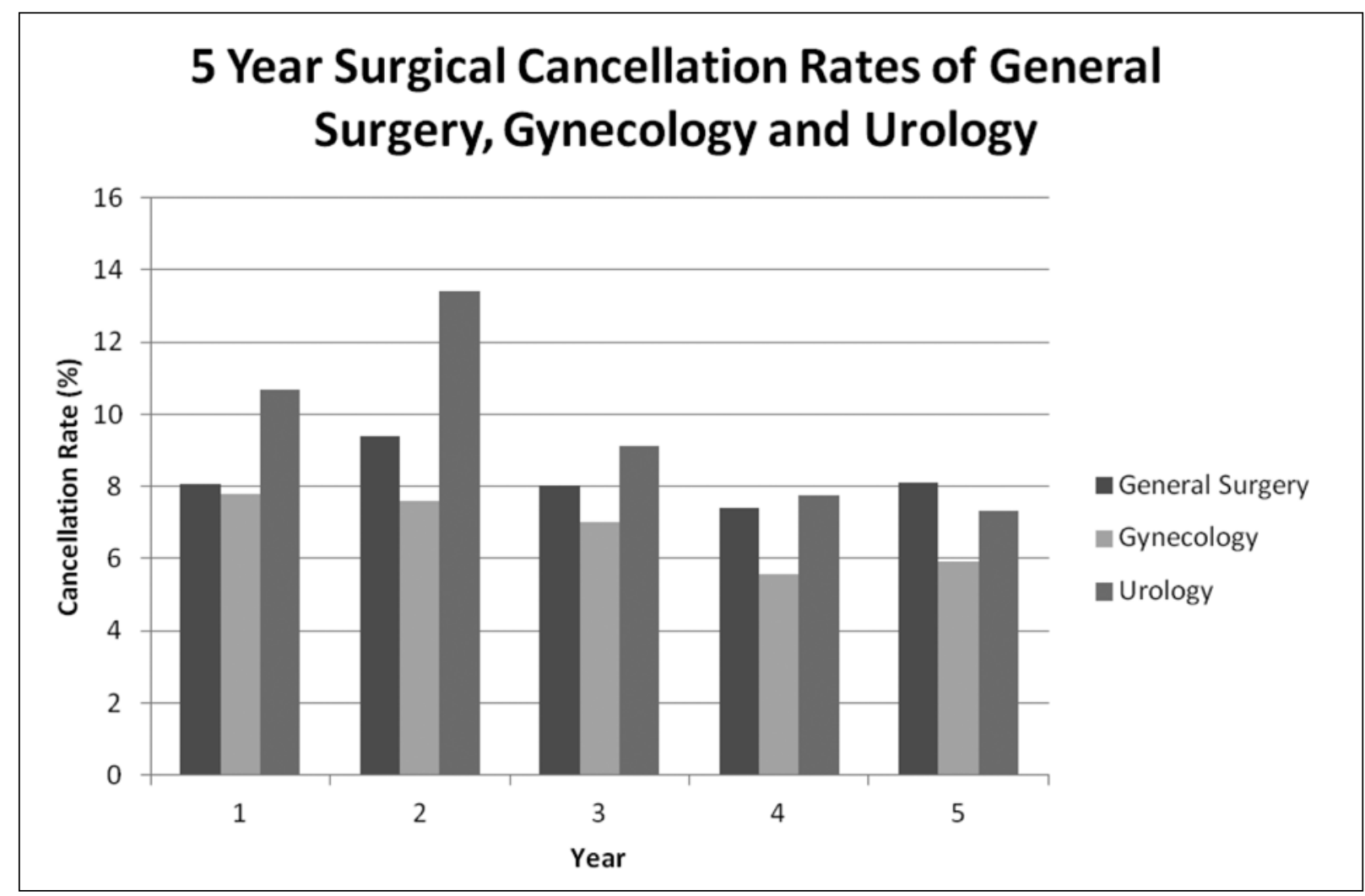

Fig. 1a. Cancellation rates for General Surgery, Gynecology and Urology services over 5-year study period (April 2005 to March 2010). 
Leslie et al.

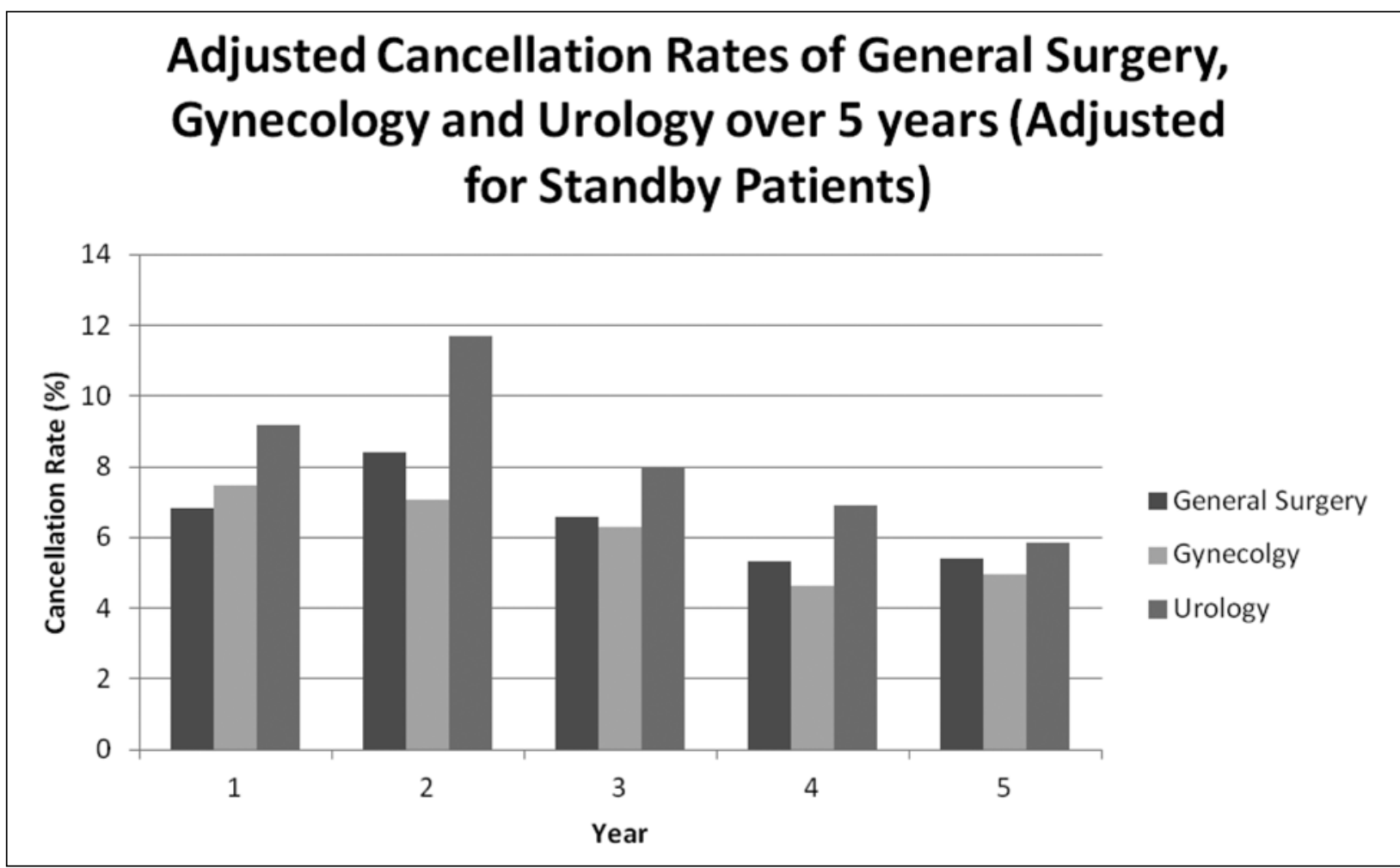

Fig. 1b. Cancellation rates for General Surgery, Gynecology and Urology services over 5-year study period (April 2005 to March 2010), adjusted for standby patients.

14 by their physician and 6 by a nurse. Interview respondents were modestly satisfied $(3.9$, mean $\pm 1.4 \mathrm{SD})$ with the perioperative process on the day of their cancelled cases; $63 \%$ agreed that it was handled appropriately. Four of the 50 patients were repeat cancellations. The average amount of time required to reschedule cases was 5 weeks, with $69 \%$ waiting less than 2 weeks. Three patients (10\%) reported that they needed to seek medical attention in the intervening time; however, 23 (80\%) believed their cancellation did not lead to any problems or a worse health outcome. Patients did report that the day-of-surgery cancellation did lead to a modest amount $(2.0 \pm 1.1)$ of additional emotional burden; $34 \%$ of patients said that the cancellation led to severe or moderate stress ( 4 and 5 on Likert scale). Similarly, financial burden was reported to be modest $(2.0 \pm 1.3)$, with $20 \%$ reporting severe or moderate financial issues encumbered by the cancellation.

\section{Table 1. Cancellation rates of oncology versus non- oncology related procedures between April 2005 and March 2010}

\begin{tabular}{lcc}
\hline & $\begin{array}{c}\text { Oncology } \\
\text { cancellation rate }\end{array}$ & $\begin{array}{c}\text { Non-oncology } \\
\text { cancellation rate }\end{array}$ \\
\hline Cancellations/procedures & $33 / 656$ & $447 / 3007$ \\
Cancellation rate (\%) & $5 \%$ & $15 \%$ \\
\hline *Rates vary significantly (Chi-Square, $p<0.0001)$. &
\end{tabular}

\section{Discussion}

In this retrospective, contemporary series of elective surgical cases, overall the cancellation rate of $8.1 \%$ was lower than previous reports, but still represents a significant barrier to timely care. Of the three surgical services reviewed, urology had the highest rate of cancellation of $9.5 \%$ over the 5 -year period of time; this confirms other reports that urology is prone to day-of-surgery cancellations. ${ }^{20-23}$ Although some of these reports categorized the reasons for cancellation differently, ${ }^{22,23}$ it appears that most cancellations were likely also do to process-related issues. The explanations for these higher cancellation rates are likely multifactorial, but may include the variable case mix of urological practice often weighted to non-oncologic, non-urgent cases in an older, potentially more comorbid, patient population. Furthermore, persistent underestimation and turnover time by surgeons may bias against services with higher percentage shorter and generally stable duration of OR times. ${ }^{20}$

The prospectively collected, administrative data on cancelled cases in many institutions would appear to be limited in their ability to provide robust information to highlight problem areas and direct policy to reduce late surgical cancellations. ${ }^{20}$ Several reports categorize reasons for surgical cancellations from data collected within the institution to identify modifiable factors, although none are extensively 


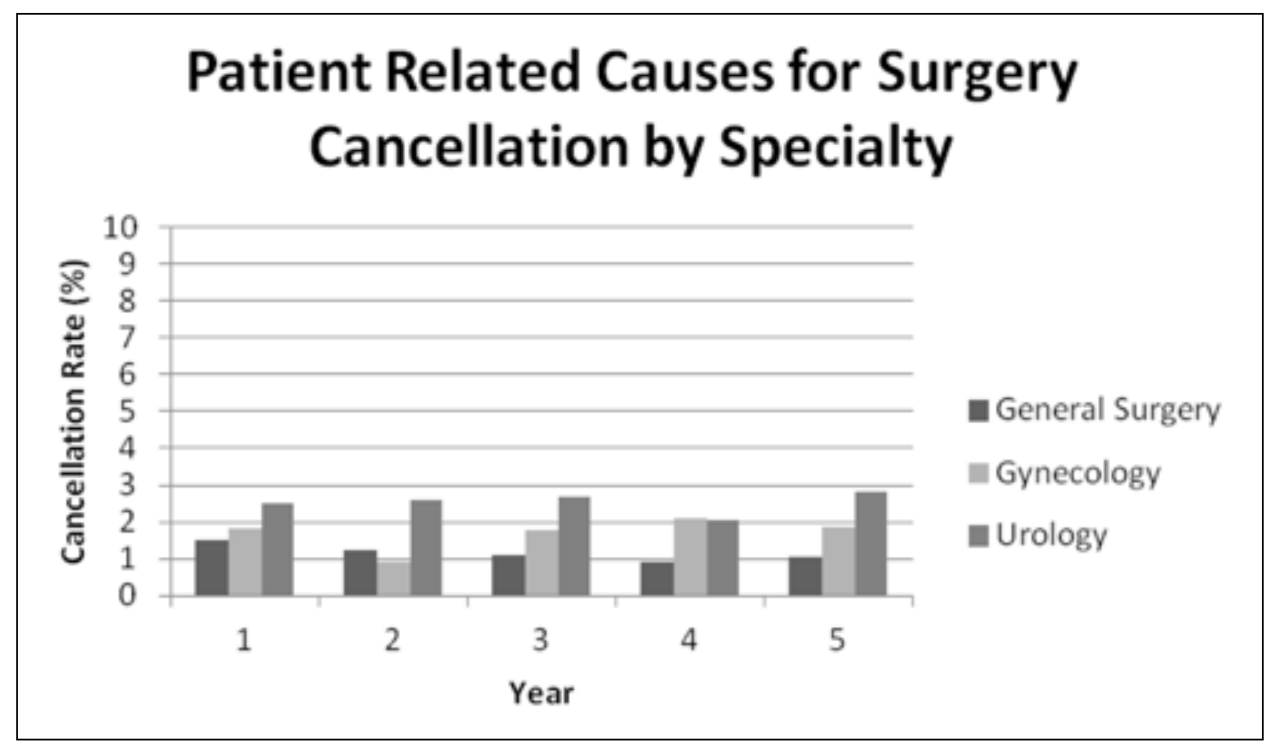

Fig. 2. Percentage of patient-related causes for cancellation of surgery. Figure displays a low average patientrelated cancellation rate of about $1.8 \%$ with minimal variation between specialty and year during study period.

validated. ${ }^{4,17-20}$ In our experience, the available data allow some categorization of reasons for cancellations into patient-, structure- and process-related factors, differences of which were sensitive to variations over time as well as between surgical specialties. Our strategy of categorization is similar to those in other reports, ${ }^{18,19}$ and it could be useful to identify potentially more modifiable reasons for cancellation.

We found that in urology, 55\% of cancellations were secondary to process-related causes. Although this categorization was retrospective and perhaps arbitrary, as most of the factors associated with cancellation are inter-related, it appeared to be sensitive to changes over time and any changes seen in cancellation rates in the urological service was mostly informed by improvements in this area. For example, cases listed on the final OR schedule as "standby" were responsible for a significant number of cancelled cases. Internal changes to the listing and management of these cases over the study period affected overall cancellation rates in the urological service. Although targeting such discrete issues are helpful, tackling each problem in the process, beginning with the initial booking to patient notification, is likely required to attain sustained quality improve-

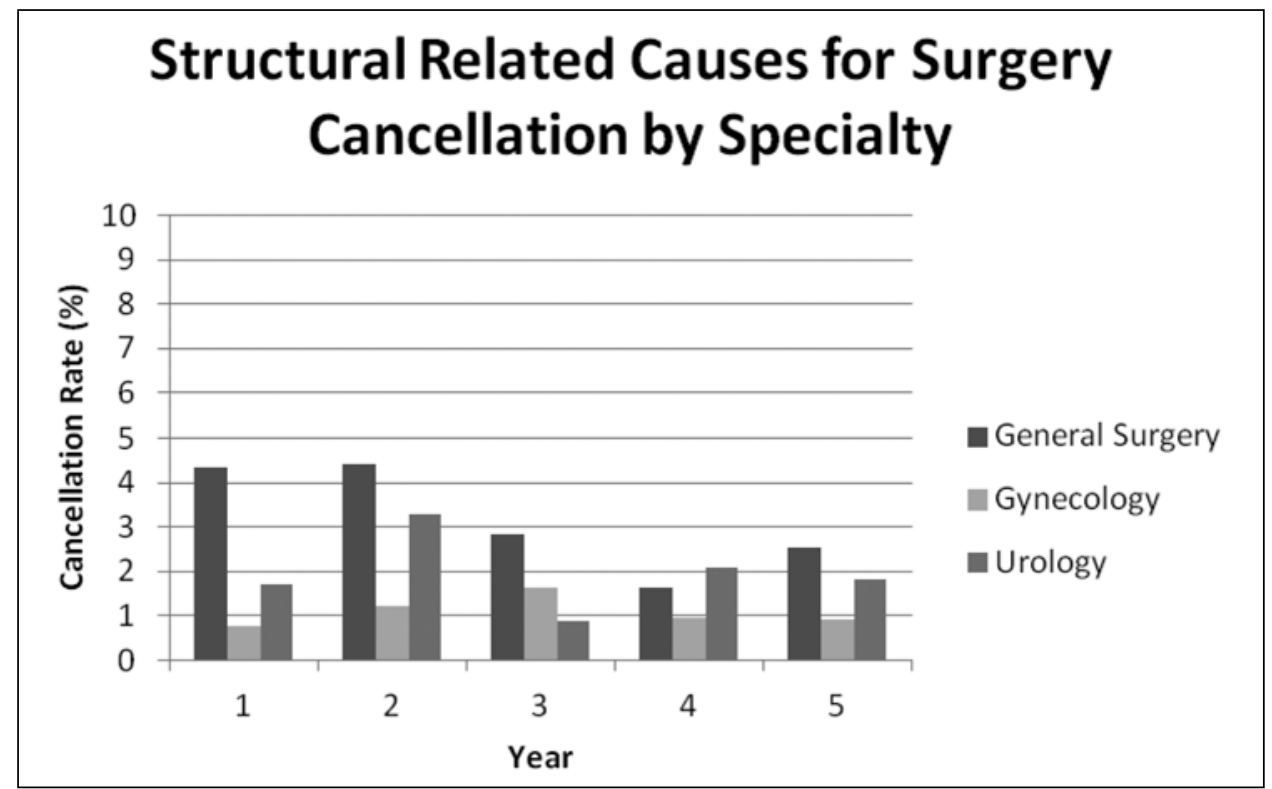

Fig. 3. Percentage of structure-related causes for cancellation of surgery by specialty per year during study period. General surgery had the highest average structure-related reasons for cancellation of surgery at $3.2 \%$. Structurerelated cancellations for Urology and Gynecology averaged $2 \%$ and $1.1 \%$, respectively, during the study period. 
Leslie et al.

\begin{tabular}{|c|c|c|c|c|}
\hline & $\begin{array}{l}\text { General } \\
\text { surgery }\end{array}$ & Gynecology & Urology & Total \\
\hline Patient & $93(13 \%)$ & $96(24 \%)$ & $113(26 \%)$ & 302 \\
\hline Process & $403(57 \%)$ & $249(61 \%)$ & $238(55 \%)$ & 890 \\
\hline Structural & $213(30 \%)$ & $60(15 \%)$ & 79 (18\%) & 352 \\
\hline Total & 709 & 405 & 430 & 1544 \\
\hline
\end{tabular}

ment. We strongly feel that there is potential to better monitor cancellations to improve OR utilization. Unfortunately, the available administrative data did not allow for more in-depth description and assessment of other process-related issues; the extensive chart review and patient interviews only resulted in further insight into reasons for cancellation in $28 \%$ of cases (data not shown). Chart review data were not used to change categorization coding when conflicting charting was found, however this was a rare event. We suggest that each surgical cancellation should be considered an adverse incident with more robust, prospective data collection and frequent reporting given the apparent fluidity of rates over time.

Day-of-surgery cancellations may have emotional and economic impacts on patients; qualitative and quantitative interviews from our study and results from other studies confirm this finding. ${ }^{7-10}$ However, it is interesting that most patients did not feel that the cancellation was associated with further health problems or a worse outcome. Specific areas of improvement identified from the patient interviews were communication between OR staff and patients, as well as the timing of OR rescheduling, particularly in the context of financial issues as well as prolonged wait times for nononcological urological cases.
As with all survey studies a limitation of this data is selection bias. The fact that we could not reach $26 \%$ of patients (without valid contact information) and another $16 \%$ (unreachable or chose not to participate) may add to the bias of our results. A further limitation of our study was the cancellation codes. They were often inadequate and did not account for cancellations that were multifactorial, nor did they identify the root cause of the cancellation. For example, "surgery running late" could be due to delays in the post-anaesthesia care unit earlier in the day. We need to start identifying the root causes of cancellations and document whether they were avoidable or not.

\section{Conclusion}

We believe that every cancellation should be considered a failure of the system and, consequently, an incident report should be filed for each cancellation. This failure would require a detailed note with an explanation of the potentially multiple reasons for cancellation with input from multiple sources rather than a single code. These results would be reviewed by the OR manager and the key stakeholders to ensure constructive change occurs to reduce inefficiencies in the system.

Acknowledgments: We would like to acknowledge the work of Kathleen Wattie Barnett in direction and data retrieval as well as the financial support of the John Franklin Kidd Studentship Fund.

Competing interests: None declared.

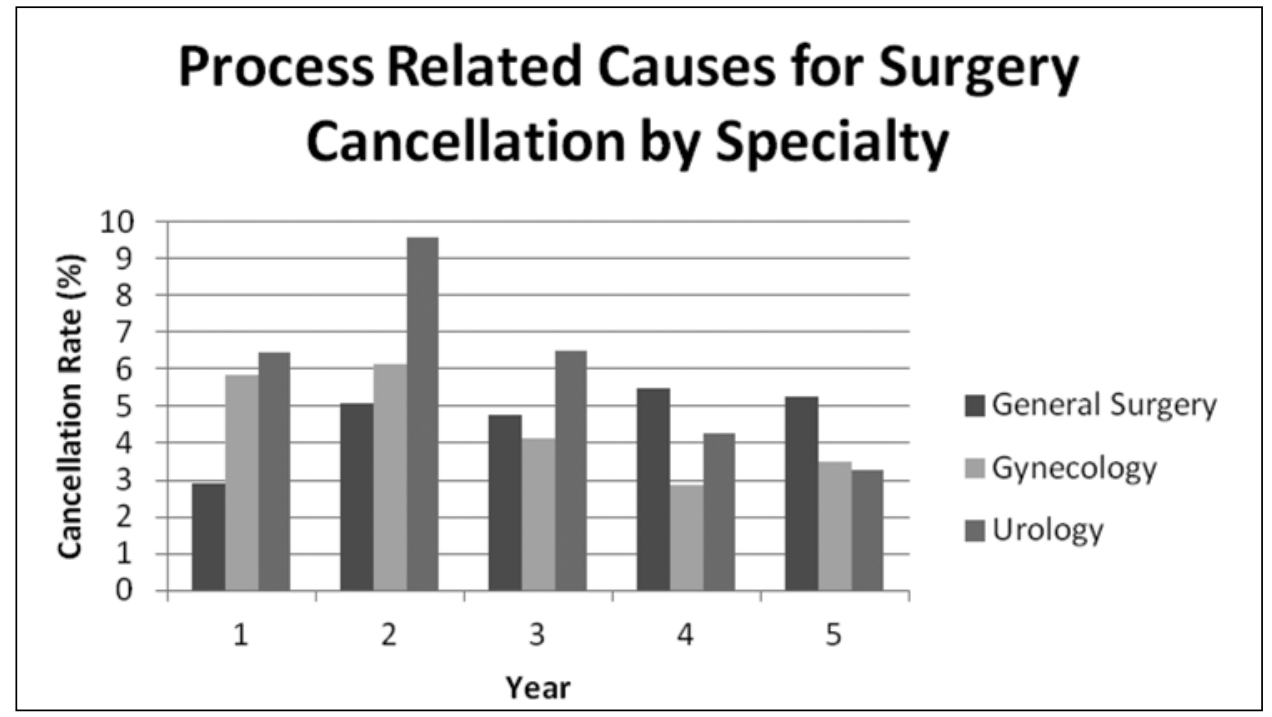

Fig. 4. Percentage of process-related causes for cancellation of surgery. Figure displays large variation of cancellation rate due to process related causes between specialty and year during study period. Percentage of process-related Urology cancellation varies between 3.3 and $9.6 \%$, averaging $6.0 \%$. Process related cancellations for General Surgery and Gynecology averaged $4.7 \%$ and $4.5 \%$, respectively, during the study period. 
This paper has been peer-reviewed.

\section{References}

1. Schuster M, Neumann C, Neumann K, et al. The Effect of Hospital Size and Surgical Service on Case Cancellation in Elective Surgery: Results from a Prospective Multicenter Study. Anesth Analg 2011;113:578-85.

2. Dexter F, Marcon E, Epstein RH, et al. Validation of statistical methods to compare cancellation rates on the day of surgery. Anesth Analg 2005;101:465-73. http://dx.doi.org/10.1213/01. ANE.0000154536.34258.A8

3. Donabedian A. The quality of care. JAMA 1988;260:1743-8. http://dx.doi.org/10.1001/ jama.260.12.1743

4. Sanjay P, Dodds A, Miller E, et al. Cancelled elective operations: an observational study from a district general hospital. J Health Organ Manag 2007;21:54-8. http://dx.doi.org/10.1108/14777260710732268

5. Pollard JB, Zboray AL, Mazze RI. Economic benefits attributed to opening a preoperative evaluation clinic for outpatients. Anesth Analg 1996;83:407-10.

6. Schofield WN, Rubin GL, Piza M, et al. Cancellation of operations on the day of intended surgery at a major Australian referral hospital. Med J Aust 2005;182:612-5.

7. Siemens DR, Schulze KM, Mackillop WJ, et al. A population-based study of the waiting times for prostatectomy in Ontario. Can I Urol 2005;12:2568-74.

8. Ivarsson B, Kimblad PO, Sjöberg T, et al. Patient reactions to cancelled or postponed heart operations. J Nurs Manag 2002;10:75-81. http://dx.doi.org/10.1046/i.0966-0429.2001.00293.x

9. Kawakami J, Hopman WM, Smith-Tryon R, et al. Measurement of surgical wait times in a universal health care system. Can Urol Assoc J 2008;2:597-603.

10. Tait AR, Voepel-Lewis T, Munro HM, et al. Cancellation of pediatric outpatient surgery: Economic and emotional implications for patients and their families. J Clin Anesth 1997;9:213-9. http://dx.doi. org/10.1016/S0952-8180(97)00032-9

11. Jones AR, Sandison AJ, Owen WJ. The impact of pre-clerking clinics on surgical operation cancellations: a prospective audit. Int J Clin Pract 1997;51:294-5.
12. Aimakopoulos G, Harrison R, Magnussen PA. Pre-admission clinic in an orthopaedic depart department: evaluation over a 6-month period. J $R$ Coll Surg Edinb 1998;43:178-81.

13. National Health Service. Results from the theatre programme cancelled operations diagnostic tool. London: NHS, 2002.

14. American Medical Association. Code of ethics. Appointments changes. 2001; http://www.ama-assn. org/ama/pub/physician-resources/medical-ethics/about-ethics-group/ethics-resource-center/educationalresources/federation-repository-ethics-documents-online/american-psychiatric-association.page (Accessed September 4, 2012).

15. Tung A, Dexter F, Jakubczyk $S$, et al. The limited value of sequencing cases based on their probability of cancellation. Anesth Analg 2010;111:749-56. http://dx.doi.org/10.1213/ANE.0b013e3181e66le8

16. Pollard JB, Olson L. Early outpatient preoperative anesthesia assessment: does it help to reduce operating room cancellations? Anesth Analg 1999;89(2):502-5. http://dx.doi.org/10.1213/00000539199908000-00048

17. Haana V, Sethuraman K, Stephens L, et al. Case cancellations on the day of surgery: an investigation in an Australian paediatric hospital. ANZ J Surg 2009;79:636-40. http://dx.doi.org/10.1111/j.14452197.2009.05019.x

18. Aguirre-Cordova J, Chavez-Vazquez G, Huitron-Aguillar $G$, et al. Why is surgery cancelled? Causes, implications, and bibliographic antecedents. Gac Med Mex 2003;139:545-51.

19. Jiménez $A$, Artigas $C$, Elia $M$, et al. Cancellations in ambulatory day surgery: Ten years observational study. Ambul Surg 2006;12:119-23. http://dx.doi.org/10.1016/i.ambsur.2005.09.002

20. Schofield WN, Rubin GL, Piza M, et al. Cancellation of operations on the day of intended surgery at a major Australian referral hospital. Med J Aust 2005; 182:612-5.

21. Lacqua M, Evans J. Cancelled elective surgery: an evaluation. Am Surg 1994;60:809-11.

22. Chiu CH, Lee A, Chui PT. Cancellation of elective operations on the day of intended surgery in a Hong Kong based hospital: point, prevalence and reasons. Hong Kong Med J 2012;18:5-10.

23. Argo JL, Vick CC, Graham LA, et al. Elective surgical case cancellation in the Veterans Health Administration system: identifying areas for improvement. Am J Surg 2009;198:600-6. http://dx.doi.org/10.1016/i. amisurg.2009.07.005

Correspondence: Dr. D. Robert Siemens, Department of Urology, Kingston General Hospital, Queen's University, Kingston, 0N K7L 2V7; fax: 613-545-1970; siemensı@kgh.kari.net

\begin{tabular}{|c|c|c|c|c|c|}
\hline \multicolumn{6}{|c|}{$\begin{array}{l}\text { Appendix 1. Categorization of reason for cancellations of surgery into: patient-, process-, and structure-related reasons. The } \\
\text { frequency of cancellation by reason over the } 5 \text { year study period has been included. }\end{array}$} \\
\hline Group 1: Patient & Frequency & Group 2: Process & Frequency & Group 3: Structural & Frequency \\
\hline Case Aborted Post Anesthesia & 1 & Administration Reconciliation & 1 & Crisis Cancellation & 27 \\
\hline $\begin{array}{l}\text { Done previously as an } \\
\text { Emergency }\end{array}$ & 6 & Anaesthesiologist Late & 3 & Emergency A Case & 33 \\
\hline Medical reasons & 4 & Cancelled by Institution & 3 & $\begin{array}{l}\text { Emergency Case inserted } \\
\text { Other Service }\end{array}$ & 14 \\
\hline Patient did not show & 16 & Delayed Start Time & 2 & $\begin{array}{l}\text { Emergency Case inserted } \\
\text { Same Service }\end{array}$ & 153 \\
\hline Patient Expired & 2 & Incorrectly booked Surgeon Office & 19 & Equipment Broken & 7 \\
\hline Patient not available & 27 & Insufficient Work Up & 8 & No Bed & 49 \\
\hline Patient Refused Procedure & 48 & Moved to another date surgeon office & 16 & No Step down Bed & 54 \\
\hline Patient too ill/ not fit for surgery & 175 & No Anesthesia Available & 24 & No ICU bed & 8 \\
\hline \multirow[t]{10}{*}{$\begin{array}{l}\text { Surgery no longer needed/ } \\
\text { inoperable }\end{array}$} & 23 & Office cancelled & 9 & Room unavailable & 5 \\
\hline & & Operating Room/PACU Staff Problems & 15 & Weather & 2 \\
\hline & & Patient not NPO & 21 & & \\
\hline & & Room on hold/other room running late & 10 & & \\
\hline & & Room running late & 71 & & \\
\hline & & Scheduling error OR office & 3 & & \\
\hline & & Standby patient cancelled & 266 & & \\
\hline & & Surgeon ill or not able to operate & 2 & & \\
\hline & & Surgeon overbooked & 11 & & \\
\hline & & Surgery running late & 406 & & \\
\hline Total & 302 & Total & 890 & Total & 352 \\
\hline
\end{tabular}

